\title{
Entstehung und Handhabung von Vorgehensmodellen zur Software-Auswahl und Software-Einführung in der IV-Beratung
}

\author{
Volker Nissen \\ Institut für Wirtschaftsinformatik, FG WI-2, \\ Technische Universität Ilmenau \\ volker.nissen@tu-ilmenau.de
}

\section{Einleitung und Forschungsfragen}

In diesem Beitrag wird auf empirischem Wege der Frage nachgegangen, wie Vorgehensmodelle zur Auswahl bzw. Einführung von Unternehmenssoftware in Beratungsunternehmen entstanden sind und praktisch gehandhabt werden. Unter einem Vorgehensmodell soll dabei die Folge aller Aktivitäten verstanden werden, die zur Durchführung eines Projektes erforderlich sind (Stahlknecht und Hasenkamp 2005, S. 215). Die Aktivitäten bzw. Aufgaben werden in gegeneinander abgegrenzten Phasen zusammengefasst. Diese enden jeweils mit definierten Meilensteinen, d. h. Entscheidungs- bzw. Genehmigungspunkten bezüglich der definierten Zwischenergebnisse (Seibt 1997b, S. 431-432). Vorgehensmodelle dienen einer besseren Prozesskontrolle, indem die Gesamtkomplexität des Projektes reduziert, die Transparenz des Prozesses verbessert und somit eine Überprüfung der Termin-, Kosten-, Qualitäts- und Leistungsziele ermöglicht wird. Außerdem werden die Koordination des Projektteams und die frühzeitige Fehlererkennung erleichtert.

Da die Gewährleistung einer qualitativ hochwertigen Dienstleistung ein zentraler Wettbewerbsfaktor ist (Bruhn 2008, S. 3), sollten, laut Mieschke, Beratungsunternehmen Vorgehensmodelle für einzelne Projekttypen bzw. Beratungsthemen mit dem Ziel der Qualitätssteigerung anwenden (Mieschke 2004, S. 105).

Die Informationsverarbeitung von Unternehmen ist ein primäres Tätigkeitsfeld von 21,6\% der ca. 13.600 in Deutschland 2008 tätigen Unternehmensberatungen (BDU 2009). Es soll hier kurz als IV-Beratung bezeichnet werden und ist stärker operativ ausgerichtet als die Strategieberatung (Nissen und Kinne 2008, S. 90). Für 
einige Tätigkeitsbereiche der IV-Beratung existieren in der Literatur empfohlene Vorgehensmodelle oder Beschreibungen von Arbeitsfolgen. Die Fragen der Entstehung und tatsächlichen Nutzung von Vorgehensmodellen in Beratungshäusern scheinen jedoch bislang noch nicht untersucht worden zu sein. Dies soll empirisch für den deutschen Beratungsmarkt geklärt werden. Hierzu werden nachfolgend die Ergebnisse einer ersten, im Umfang allerdings beschränkten qualitativen Fallstudienanalyse vorgestellt (Nissen und Simon 2009). Deren Ergebnisse sollen im zweiten Schritt durch eine breitere, quantitative Erhebung abgesichert werden, die derzeit vorbereitet wird.

Der Beitrag ist wie folgt strukturiert: Zunächst werden typische Aufgabenstellungen der IV-Beratung in kurzer Form vorgestellt. Im Abschnitt 3 werden diese dann in drei von Maister (2003) vorgeschlagene Komplexitätskategorien (Procedure, Grey Hair und Brain Projekte) eingeordnet. Die Auswahl bzw. Einführung von Unternehmenssoftware fallen demnach in die Kategorie der Procedure-Projekte. Damit erscheinen sie besonders geeignet für den Einsatz weitgehend standardisierter Vorgehensmodelle. In Abschnitt 4 wird daher empirisch untersucht, wie in diesen beiden Anwendungsbereichen Vorgehensmodelle in IV-Beratungsunternehmen entstanden sind und aktuell verwendet werden. Der Beitrag schließt mit einem kurzen Fazit und Ausblick auf weiteren Forschungsbedarf.

\section{Typische Aufgabenstellungen der IV-Beratung}

Anhand einer umfassenden Analyse der Internetseiten von 30 etablierten IV-Beratungsunternehmen konnte ein vielfältiges Angebot an Beratungsleistungen identifiziert werden. Die am häufigsten genannten Kernaufgaben der IV-Beratung werden nachfolgend kurz dargestellt.

- Software-Auswabl: Das Beratungsfeld der Software-Auswahl umfasst den gesamten Prozess der Auswahl einer für die individuellen Unternehmensziele bestmöglich geeigneten Software - in der Regel Unternehmenssoftware ${ }^{1}$. Die Auswahl des „besten“ Software-Anbieters bzw. Vertriebspartners ist neben der Auswahl des Produktes ebenfalls Bestandteil des Aufgabenfeldes. Dieser Aspekt ist darum von großer Bedeutung, weil die sich dem Auswahlprozess anschließende Software-Einführung oft gemeinsam mit diesem Unternehmen durchgeführt wird.

- Software-Einfuibrung: Aus Sicht des Beratungshauses besteht die Aufgabenstellung der Software-Einführung vornehmlich darin, eine betriebliche Standardsoftware „(..) zielorientiert und toolgestützt unter maßgeblicher Beteiligung des Kunden einzuführen mit dem Ziel, einen optimalen Nutzen langfristig zu ermöglichen“ (Gabriel und Lohnert 2001, S. 182). Hierzu gehören häufig auch Aufgabenstellungen der betrieblichen Reorganisation und Prozessberatung.

\footnotetext{
${ }^{1}$ Damit sind v.a. ERP-Systeme und Warenwirtschaftssysteme gemeint (Becker et al 2007, S. 2).
} 
- Informationsarcbitekturberatung: Die Informationsarchitektur bildet als „das grundlegende logische Modell der Informationsinfrastruktur" die Basis für die weiteren Architekturkomponenten und ist dabei „das Ergebnis der (...) unternehmensweit erfassten, evaluierten und gegliederten Informationsnachfrage bzw. der zu ihrer Befriedigung erforderlichen Informationsversorgung (...)“ (Heinrich et al 2004, S. 319). In diesem Beratungsfeld werden vielfältige Leistungen angeboten. Sie reichen von der Beratung zur Gestaltung einer Informationsarchitektur über die Unterstützung bei der Festlegung einheitlicher Architekturregeln bis hin zur Optimierung der Architektur einzelner Softwarelösungen.

- IT-Sicherbeitsberatung: Gemäß der Capgemini-Studie „IT-Trends 2008“ ist für viele Unternehmen die IT-Sicherheit das wichtigste IT-Thema (Capgemini 2008, S. 7, 26). Um diesbezüglichen Gefahren zu begegnen, ist ein ganzheitliches Informationssicherheitsmanagement durch technische, organisatorische und physische Maßnahmen erforderlich, das ein weites Betätigungsfeld für IV-Unternehmensberater bildet. Hierzu gehören insbesondere die Durchführung von Risiko- bzw. Sicherheitsanalysen zur Feststellung des Bedrohungspotentials sowie Unterstützung bei der Konzeption und Umsetzung von IT-Sicherheitskonzepten.

- IT-Integrationsberatung: Die heute in Unternehmen vorherrschenden heterogenen IT-Landschaften erschweren die durchgängige, effiziente Unterstützung von Geschäftsprozessen und führen $\mathrm{zu}$ hohen Wartungsaufwänden. Die IT-Integrationsberatung befasst sich mit allen Teilaspekten einer Verbesserung der integrierten Informationsverarbeitung im Klientenunternehmen. Dazu gehören die Prozessintegration in Bezug auf die Automatisierung der Geschäftsprozesse, die Herstellung einer einheitlichen Datenbasis im Rahmen der Datenintegration sowie die homogene Präsentation verschiedener Programme durch die Integration der Benutzerschnittstellen (Mertens 1997, S. 208). Ein typisches Beispiel für ein Integrationsprojekt ist der Aufbau eines Unternehmensportals.

- IT-Strategieberatung: Die Strategieentwicklung ist eine Teilaufgabe der strategischen IT-Planung (Heinrich et al 2004, S. 633-634). Dabei wird die IT-Strategie aus der Unternehmensstrategie abgeleitet bzw. mit dieser abgestimmt (Business-ITAlignment), um den Wertbeitrag der IT für das Unternehmen zu erhöhen. Die Aufgabe der Unternehmensberatung ist hierbei die umfassende Unterstützung des Klienten bei der Entwicklung und Umsetzung der IT-Strategie.

- Beratung zur IT-Organisation: Dieses Beratungsfeld ist eng mit dem der IT-Strategie verknüpft. Unter Beachtung der strategischen Vorgaben wird die IT-spezifische Aufbau- und Ablauforganisation des Klientenunternehmens entwickelt. Zum einen können die Berater dem Klienten eine Anleitung bei der hierarchischen Einordnung und internen organisatorischen Gliederung der Organisationseinheit „Informationsverarbeitung“ geben. Und zum anderen beschäftigt sich die Beratung mit den ,organisatorisch geregelten Prozesse(n) der Beschaffung, der Erstentwicklung, des Betriebs, der Wartung und der Weiterentwicklung der verschie- 
denen Arten von Systemen“ (Seibt 1997a, S. 42). Von großer Bedeutung sind Referenzmodelle und Methoden des IT-Servicemanagements, wie etwa ITIL.

- IT-Outsourcing-Beratung: IT-Outsourcing bezeichnet die teilweise oder komplette Übertragung von Aufgaben und Ressourcen der Informationsverarbeitung eines Unternehmens an einen oder mehrere rechtlich unabhängige Dienstleister. Hier muss betont werden, dass die Aufgabe der IV-Beratung sich nur auf die Unterstützung der Klienten im Rahmen der Vorbereitung und Durchführung eines Outsourcing-Vorhabens beziehen. IV-Beratung besteht jedoch nicht darin, die Outsourcing-Leistungen selbst zu erbringen.

\section{Kategorisierung der Aufgabenstellungen nach Komplexität}

David Maister hat eine Kategorisierung von Projekttypen der Unternehmensberatung vorgeschlagen, die inzwischen weite Verbreitung in der beratungsbezogenen Literatur gefunden hat. Er unterscheidet zwischen Brain, Grey Hair und Procedure Projekten (Maister 2003, S. 4-5):

- Brain Projekte: Die hier zu lösende Kundenaufgabe ist neu und von extremer Komplexität. Sie erfordert höchstes methodisch-fachliches Wissen und professionelle Fähigkeiten. Als Kernanforderungen solcher Projekte an die Berater werden Kreativität, Innovation und Pionierleistungen bei neuen Ansätzen, Konzepten und Techniken genannt. Der Klient kauft hier von der Beratung vor allem überragende intellektuelle Fähigkeiten ein, um seine Probleme zu lösen. Daher können solche Projekte nur von besonders hoch qualifizierten, intellektuell herausragenden Beratern durchgeführt werden.

- Grey Hair Projekte: Diese Projekte verlangen eine individuelle Lösung, sind jedoch in ihren Anforderungen an Kreativität und Innovativität geringer einzuschätzen als Brain Projekte. Die zu lösende Aufgabenstellung ist im Grundsatz bekannt und Lösungsansätze aus anderen Projekten können darauf übertragen werden. Klienten achten bei der Beauftragung eines Beratungsunternehmens vor allem auf nutzbare Erfahrungen und Vorwissen aus früheren Projekten sowie das sich daraus ergebende Urteilsvermögen der Berater.

- Procedure Projekte: Der hier zu bearbeitende Problemtyp ist gut bekannt und die einzelnen Lösungsschritte liegen weitgehend fest. Klienten könnten die damit verbundenen Aufgaben oft selbst lösen, haben aber nicht die erforderlichen Ressourcen, um dies mit vergleichbarer Effizienz wie eine Unternehmensberatung zu tun.

Ein Vorschlag, wie die genannten Kernaufgaben der IV-Beratung anhand von Komplexität und Wiederholungscharakter den Kategorien von Maister zugeordnet werden können, ist in Tabelle 1 dargestellt. ${ }^{2}$ Aufgabenstellungen aus der Kategorie

\footnotetext{
2 Die Einordnung beruht auf mehr als 10 Jahren eigener Erfahrung in der IV-Beratung sowie ergänzenden Experteninterviews.
} 
„Procedure Projekt" erscheinen besonders geeignet, um durch weitgehend standardisierte Vorgehensmodelle die Projektarbeit effizienter zu gestalten. So ist es nicht verwunderlich, dass insbesondere für die Themen Software-Auswahl und Einführung in der Beratungspraxis zahlreiche Vorgehensmodelle existieren.

\section{Tabelle 1: Einordnung von Aufgaben der IV-Beratung in Komplexitäts} kategorien

\begin{tabular}{|l|l|l|l|}
\hline $\begin{array}{l}\text { Beratungsfeld innerhalb der IV- } \\
\text { Beratung }\end{array}$ & $\begin{array}{l}\text { Brain } \\
\text { Projekte }\end{array}$ & $\begin{array}{l}\text { Grey Hair } \\
\text { Projekte }\end{array}$ & $\begin{array}{l}\text { Procedure } \\
\text { Projekte }\end{array}$ \\
\hline Software-Auswahl & & & $\mathrm{X}$ \\
\hline Software-Einführung & & $\mathrm{X}$ & $\underline{\mathrm{X}}$ \\
\hline Informationsarchitektur & $\mathrm{X}$ & $\underline{\mathrm{X}}$ & \\
\hline IT-Sicherheit & & $\underline{\mathrm{X}}$ & $\mathrm{X}$ \\
\hline IT-Integration & & $\mathrm{X}$ & \\
\hline IT-Strategie & $\mathrm{X}$ & $\mathrm{X}$ & \\
\hline IT-Organisation & & $\underline{\mathrm{X}}$ & $\mathrm{X}$ \\
\hline IT-Outsourcing & & $\mathrm{X}$ & \\
\hline
\end{tabular}

\section{Vorgehensmodelle in der IV-Beratungspraxis}

\subsection{Datengrundlage und Methodik der Untersuchung}

Die unten dargestellten Ergebnisse sind Teil einer empirischen Untersuchung unter IV-Beratungsfirmen in Deutschland. Dabei wurde insbesondere auch untersucht, wie die für Software-Auswahl und -Einführung mehrheitlich in der Praxis eingesetzten Vorgehensmodelle strukturiert sind (im Sinne von common bzw. best practices). Auf diesen Teil der Ergebnisse wird hier jedoch aus Platzgründen nicht näher eingegangen. ${ }^{3}$

Die Befragung fand in Form von Leitfaden-Interviews mit überwiegend offener Frageformulierung am Telefon statt. Die Gesprächszeit variierte dabei zwischen 45 Minuten und zwei Stunden, wobei die Interviewpartner erfahrene Berater mit langer, einschlägiger Berufspraxis waren. Diese qualitative Vorgehensweise erlaubt im Unterschied zur anonymen Befragung mittels Fragebogen auch Rückfragen von beiden Gesprächsparteien, so dass Missverständnisse ausgeräumt und Hintergründe zu Antworten geklärt werden können.

${ }^{3}$ Siehe hierzu Nissen und Simon (2009). 
Die Anzahl der untersuchten Firmen war methodenbegründet im Vergleich zu großzahligen Fragebogen-basierten Studien eher klein. Zunächst erfolgte im Sinne einer Vorab-Festlegung die Erstellung einer Samplestruktur, die eine begründete Stichprobenbildung erlaubte (Mayer 2006, S. 38-39). Als Schichtungsmerkmal kam die Unternehmensgröße zum Einsatz, wobei in kleine $(<20$ Mitarbeiter), mittelgroße (20 - 500 Mitarbeiter) und große $(>500$ Mitarbeiter) Unternehmen differenziert wurde. Außerdem wurde die Befragung auf den Einsatz von Vorgehensmodellen bei der Software-Auswahl und -Einführung beschränkt, da hier eine weite Verbreitung in der Praxis vorliegt. Es nahmen je fünf kleine, fünf mittelgroße und vier große Unternehmen an unseren Leitfadeninterviews teil $(\mathrm{n}=14)$.

Für die anonyme Auswertung wurden die Interviews elektronisch aufgezeichnet und im Anschluss an deren Durchführung transkribiert. Die Transkription erfolgte aufgrund der begrenzten zeitlichen Ressourcen jedoch nicht wortgetreu, sondern überwiegend sinngemäß.

Im Folgenden wird auf eine generelle Differenzierung der beiden betrachteten Beratungsfelder Software-Auswahl und Software-Einführung verzichtet. Dieses Vorgehen erscheint begründet, da die Angaben zur Entstehung und Handhabung von Vorgehensmodellen in beiden Bereichen weitgehend übereinstimmten. Nach dem Eindruck aus den Interviews hängt außerdem der praktische Umgang mit Vorgehensmodellen vor allem von der Unternehmenskultur des jeweiligen Beratungshauses und weniger vom Thema ab.

Eine Unterscheidung der befragten Unternehmen nach Größe erfolgt dort, wo diese einen hinsichtlich der Ergebnisse differenzierenden Faktor darstellt.

\subsection{Ergebnisse und Diskussion zur Entstehung der Vorgehensmodelle}

\subsubsection{Entwicklungszeitpunkt}

Alle befragten IV-Beratungsunternehmen setzen in mindestens einem der beiden betrachteten Anwendungsfelder Vorgehensmodelle ein. Es gibt drei Beratungshäuser, die ihre Vorgehensmodelle bereits seit mindestens 15 Jahren anwenden (Abb. 1). In sechs Beratungshäusern werden die Modelle seit 10 - 15 Jahren angewandt und vier Berater gaben an, dass das jeweilige Vorgehensmodell seit 5 - 9 Jahren im Einsatz ist. Ein Unternehmen machte hierzu keine Angaben. Diese im Durchschnitt recht langen Anwendungszeiträume belegen, dass die Beratungsfelder der Software-Auswahl und -Einführung zu den klassischen Kernaufgaben der IVBeratung zählen und heute als Procedure-Projekte kategorisiert werden können. 


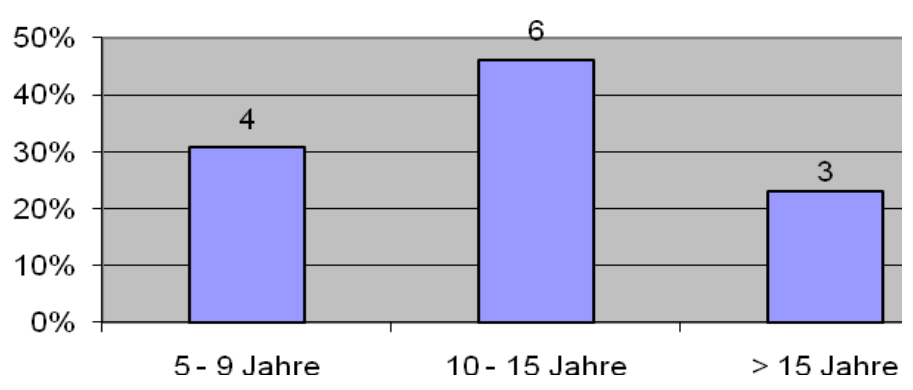

Abbildung 1: Anwendungsdauer der Vorgehensmodelle $(n=13)$

\subsubsection{Modellursprung und Entwicklungsprozess}

Zur Entwicklung von Vorgehensmodellen stehen den Unternehmen hauptsächlich zwei Methoden zur Verfügung. Entweder werden die in den durchgeführten Projekten gewonnenen Erfahrungen erfasst und in einem Modell zusammenfassend aufbereitet. Oder die Methodenverantwortlichen bedienen sich externer Quellen und adaptieren diese in einem eigenen Vorgehensmodell. Dabei kann es sich um bereits etablierte Vorgehensweisen, internationale Standards oder wissenschaftliche Literatur handeln. Welche dieser Möglichkeiten die befragten Beratungshäuser für die Entwicklung der Vorgehensmodelle nutzten, zeigt Abb. 2. Aus diesen Ergebnissen lässt sich folgern, dass das in den Projekten erlangte Wissen eine wertvolle Basis für die Erstellung von internen Vorgehensmodellen darstellt. Daneben werden externe Best Practices wie die ASAP-Methode der SAP AG ausgewertet.

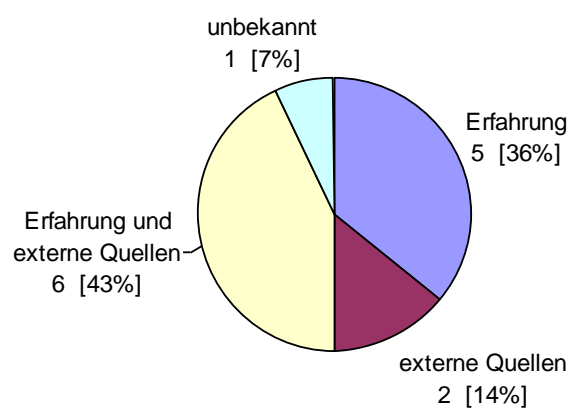

Abbildung 2: Ursprung der Vorgehensmodelle $(n=14)$

Der Entwicklungsprozess für Vorgehensmodelle unterscheidet sich je nach Unternehmensgröße außerdem deutlich. In Beratungshäusern mit wenigen Mitarbeitern findet ein weniger formalisierter, aber teils kreativerer Prozess statt, der von einem Berater neben dem Tagesgeschäft geleitet werden kann. Bei mittelgroßen und großen Unternehmensberatungen ist dagegen eine stärker systematische und formale 
Entwicklung anzutreffen. So gibt es bspw. in zwei der hier befragten großen internationalen Beratungshäuser eigene Organisationseinheiten, die sich hauptsächlich mit der Entwicklung und Verbesserung von Vorgehensmodellen befassen.

\subsubsection{Motivation für Entwicklung und Einsatz, von Vorgehensmodellen}

Die Sicherung der Beratungsqualität ist nach Meinung der befragten Berater der wichtigste Beweggrund für Vorgehensmodelle (Abb. 3). Die Vorgabe der zu leistenden Arbeitsergebnisse und Unterstützung der Qualitätssicherung im Beratungsprozess gewährleisten eine relativ einheitliche Qualität in verschiedenen Projekten. Außerdem erhöht eine strukturierte Vorgehensweise die Transparenz des Beratungsprozesses und trägt bspw. in Software-Auswahl-Projekten durch die Schaffung einer fundierten Informationsbasis zu sichereren Entscheidungen bei. Damit bieten Vorgehensmodelle auch für die Beratungsklienten eine erhöhte Sicherheit.

Das zweithäufigste Motiv für die Entwicklung und den Einsatz von Vorgehensmodellen ist ihre hervorragende Eignung, den Beratern als Arbeitsanleitung zu dienen. Wenn alle Berater mit der anzuwendenden Methode vertraut sind, wird die Kommunikation durch eine gemeinsame Grundlage erheblich erleichtert. Auch für die Zusammenarbeit mit den Klientenmitarbeitern ist eine einheitliche Verständnisgrundlage bezüglich des Projektablaufes ein erfolgskritischer Faktor. Ein weiterer Vorteil wird darin gesehen, dass eine einfache und dynamische Teamzusammenstellung ermöglicht wird. Da alle Berater nach dem gleichen Modell arbeiten, können jederzeit neue Berater zum Projekt hinzugezogen werden, ohne dass subjektive Arbeitsweisen abgestimmt werden müssen und lange Teamfindungsphasen von Nöten sind. Dieser Aspekt spielt insbesondere bei internationalen Beratungshäusern eine wichtige Rolle, weil somit globale Teams gebildet werden können. Letztlich bilden Vorgehensmodelle zudem die Grundlage für eine einheitliche und hochwertige interne Ausbildung der neuen Berater.

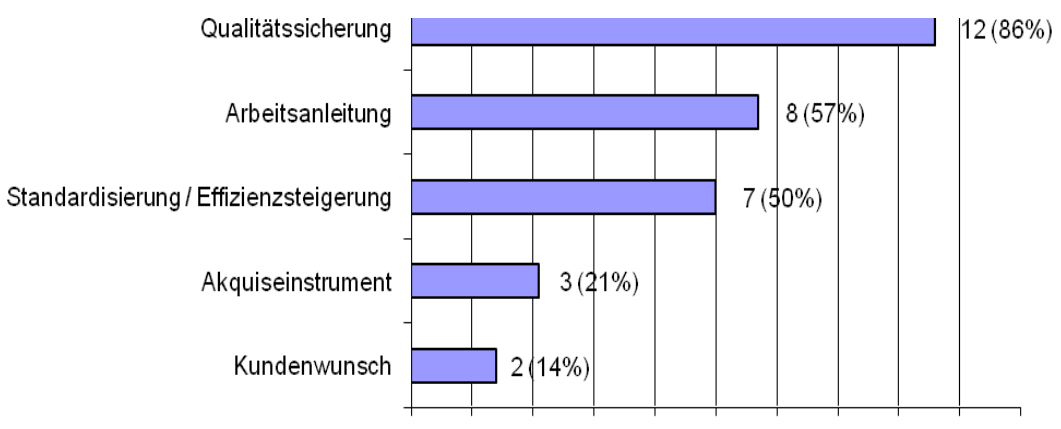

$0 \% \quad 10 \% \quad 20 \% \quad 30 \% \quad 40 \% \quad 50 \% \quad 60 \% \quad 70 \% \quad 80 \% \quad 90 \% 100 \%$

Abbildung 3: Motivation für Entwicklung/Einsatz der Vorgehensmodelle $(n=14$, Mehrfachnennungen möglich) 
Ebenfalls noch von erheblicher Bedeutung ist die Möglichkeit, Beratung hinsichtlich des Ablaufes zu standardisieren und so eine Effizienzsteigerung in ProcedureProjekten zu erreichen. So enthalten viele Vorgehensmodelle beispielsweise standardisierte Templates, best practices und empfohlene Tools zur Unterstützung der Berater. Trotz der notwendigen Anpassungen an die Klientensituation wird dadurch eine beschleunigte und effiziente Projektabwicklung erleichtert.

\subsection{Ergebnisse und Diskussion zur Handhabung der Vorgehensmodelle}

Allein das Vorhandensein eines Vorgehensmodells ist noch keine Garantie für gute Beratungsleistungen. Wenn die Berater die Methode nicht verinnerlicht haben und sie in der Projektarbeit nicht anwenden, bietet selbst das beste Modell keinen Mehrwert. Entscheidend für den erfolgreichen Einsatz eines Vorgehensmodells sind demzufolge die (unternehmenskulturelle) Verankerung des selbigen sowie verschiedene Aspekte der Modellhandhabung in der Beratungspraxis.

\subsubsection{Freiwilligkeit der Anwendung von Vorgehensmodellen}

Die Befragung der Berater ergab folgende Verteilung: in neun der befragten Unternehmen (64\%) ist die Anwendung der Vorgehensmodelle vorgeschrieben; in den restlichen fünf Unternehmen $(36 \%$ verlässt man sich auf die freiwillige Modellnutzung durch die Berater. Größere Unternehmen schreiben die Verwendung der eigenen Vorgehensmodelle eher vor als kleine (Abb. 4).

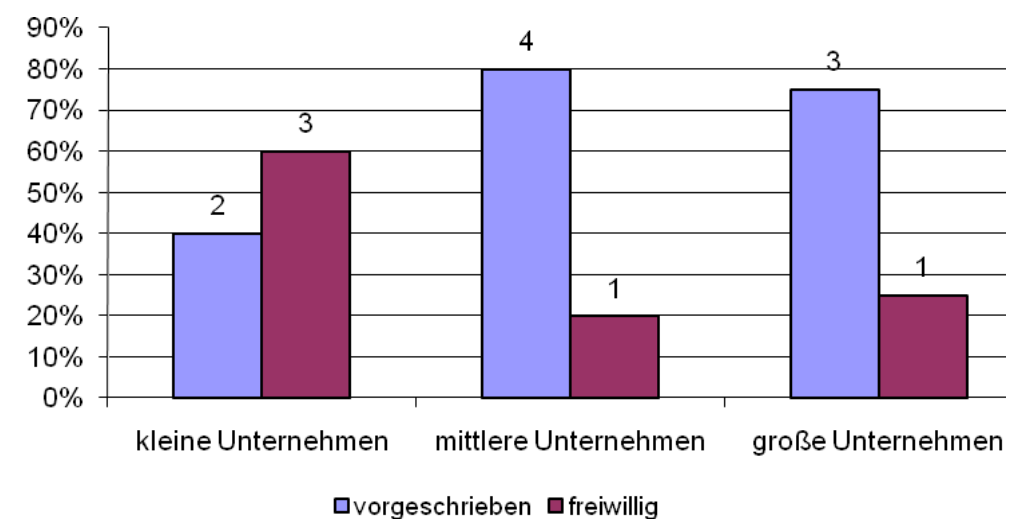

\section{Abbildung 4: Freiwilligkeit der Modellanwendung ( $n=14)$}

Die Anwendungsvorschrift sollte aber nicht als „unbedingter Zwang“ missverstanden werden. Die Berater sind zwar grundsätzlich angehalten, sich an den vorgegebenen Projektablauf zu halten. Aber zum einen wird das konkrete Vorgehen im Projekt immer an die aktuelle Situation des Klienten angepasst, was zum Beispiel dazu führen kann, dass das Modell nicht vollständig angewandt wird. Zum 
anderen haben viele Klientenunternehmen eigene Vorgehensmodelle, nach denen sich die Berater richten müssen. In solchen Fällen versucht man, das Vorgehensmodell des Beratungshauses anzupassen oder ergänzend einzusetzen.

\subsubsection{Dokumentation}

Insgesamt sind die Vorgehensmodelle in neun der befragten Beratungshäuser vollständig dokumentiert und somit expliziert. Aus der Abb. 5 ist zu erkennen, dass in ausnahmslos allen großen Unternehmensberatungen diese Form der Dokumentation vorherrscht. Dabei sind die Modelle bspw. in einer Datenbank oder im Intranet gespeichert, können von jedem Berater eingesehen und bei Bedarf auf den eigenen PC herunter geladen werden. Außerdem ist die Methode in vielen Fällen mit Templates, best practices und Projektberichten hinterlegt. In fünf der befragten Beratungen - hauptsächlich kleine Unternehmen - wurden die Vorgehensmodelle nur zum Teil expliziert. Die Formen dieser Dokumentation variieren von einer groben Arbeitsanleitung, über Templates für Arbeitsergebnisse oder Projektberichte in einer Datenbank bis hin zu einer Musterpräsentation oder der Darstellung des Modells auf der eigenen Internetseite. Der hauptsächliche Grund für diesen geringeren Explizierungsgrad ist bei den kleinen Beratungsfirmen darin zu sehen, dass die dortigen Berater durch die Eigenentwicklung ihres Modells und ihr Erfahrungswissen eigentlich keine schriftliche Ausarbeitung benötigen. Die Dokumentation erfolgt dann beispielsweise zu Marketingzwecken.

Die Beratungen, die ihre Vorgehensmodelle vollständig schriftlich dokumentiert haben, nutzen diese auch im Sinne der Wissensvermittlung zur Aus- oder Weiterbildung der Berater. Fünf Interviewpartner (zwei aus mittelgroßen und drei aus großen Beratungshäusern) gaben an, dass zu diesem Zweck Schulungen und Trainings anhand des Vorgehensmodells durchgeführt werden. Außerdem können die Vorgehensmodelle zur Unterstützung weiterer Bausteine des Wissensmanagements eingesetzt werden. So trägt die Speicherung von Projekterfahrungen und best practices sowohl zum Wissensaufbau als auch zur langfristigen Wissensbewahrung bei. Der mögliche Zugriff auf das Modell im Intranet fördert zusätzlich die Wissensverteilung unter den Beratern. In der Beratungspraxis spielt das individuelle, implizite Wissen der einzelnen Berater jedoch weiterhin eine wichtige Rolle für den Beratungserfolg. 


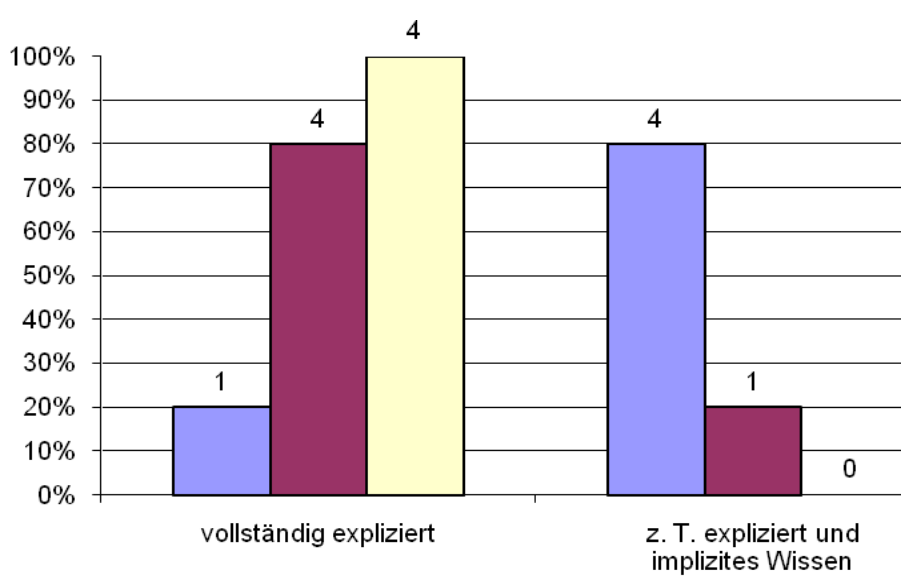

口kleine Unternehmen amittlere Unternehmen agroße Unternehmen

Abbildung 5: Dokumentation der eigenen Vorgehensmodelle $(n=14)$

Diejenigen Beratungshäuser, die ihr Vorgehensmodell nur zum Teil expliziert haben, folgen stärker einer Personalisierungsstrategie im Wissensmanagement.

\subsubsection{Zertifizierung}

Die Sicherung der Beratungsqualität wurde von den Interviewpartnern als ein wichtiger Beweggrund zur Erstellung und Nutzung von Vorgehensmodellen genannt. Um die Qualität der eigenen Prozesse und somit indirekt die Beratungsqualität gegenüber den Klienten nachweisen zu können, bietet sich eine offizielle Zertifizierung bspw. nach DIN EN ISO 9001:2000 an.

Zwei der befragten Beratungshäuser (ein kleines, ein mittleres) haben eine solche Zertifizierung durchgeführt, während die Berater der anderen 12 Unternehmen diese Frage verneinten. Auf Nachfrage gaben zwei Berater an, dass eine Zertifizierung auch zukünftig nicht angestrebt wird. Ein Berater berichtete, dass die Zertifizierung wieder aufgegeben wurde. Als Grund gegen eine Zertifizierung wurde ein schlechtes Kosten-Nutzen-Verhältnis angeführt. Einen möglichen Ausweg zeigt die Vorgehensweise eines der großen Beratungsunternehmen auf: Die Beratungsfirma selbst besitzt zwar keine Zertifizierung, aber die einzelnen Berater können sich hinsichtlich bestimmter Standards zertifizieren lassen, was als Vorteil in der Projektakquise gesehen wird.

\subsubsection{Evaluierung und Weiterentwicklung}

Als ein weiterer wichtiger Aspekt zur langfristigen Qualitätssicherung erscheint die Evaluierung der Vorgehensweise am Ende der durchgeführten Projekte und die regelmäßige Weiterentwicklung des gesamten Modells. Die klare Mehrheit der 
befragten Beratungshäuser (11) führt eine Evaluierung bezüglich der Vorgehensweise am Ende der jeweiligen Projektdurchführung durch. Die Benennungen dieser Aktivität reichen von „Schlussbilanz“, „Abschlussmeeting“ und „de-briefing“" über „lessons learned“ oder „feedback“" bis hin zu „touch down“. Dabei werden die Projektkennzahlen, wie Zeit- bzw. Termin- und Kosteneinhaltung, überprüft, um daraus auch Rückschlüsse auf die Eignung des Vorgehensmodells zu ziehen.

Neben dieser quantitativen Kontrolle werden im Rahmen der inhaltlichen Auseinandersetzung mit dem Projektablauf Erfahrungen hinsichtlich positiver und negativer Begebenheiten gesammelt. Anhand dieser qualitativen Evaluation können ebenfalls Defizite innerhalb des Vorgehensmodells identifiziert werden. AuBerdem führen zwei Beratungshäuser nach Projektende eine Messung der Kundenzufriedenheit mittels Fragebögen bzw. Befragungen durch. Durch diese Sicht von außen können zusätzliche Anregungen aufgenommen werden.

Obwohl gerade kleinere Beratungshäuser nicht immer eine explizite Evaluation nach Projektabschluss durchführen, gaben alle Befragten an, dass eine regelmäßige Weiterentwicklung ihrer Vorgehensmodelle erfolgt. Gewöhnlich wird an dem groben Phasenschema jedoch kaum etwas verändert. Die Weiterentwicklung bezieht sich vielmehr auf Details wie die Entwicklung neuer best practices, die Standardisierung von Templates oder die Verbesserung einzelner Methoden und Tools. Die Basis dieser Maßnahmen bilden in der Regel die in den Projekten gewonnenen Erfahrungen der Berater. In einem großen internationalen Beratungshaus kann sich außerdem jeder Mitarbeiter an Ideensammlungen (sog. Brainstormings) und Abstimmungen über Verbesserungsvorschläge im Intranet beteiligen. Ferner nutzen alle Berater und Methodenverantwortlichen wissenschaftliche Literatur, Veröffentlichungen in Zeitschriften oder Seminarinhalte für die Weiterentwicklung.

\subsubsection{Relevanz der Vorgehensmodelle für die Projektakquisition}

Alle bis auf einen selbstständigen Berater gaben an, dass sie von den Klienten in der Akquisitionsphase auf ihr Vorgehensmodell angesprochen werden. Dass die Einschätzungen hinsichtlich der letztendlichen Relevanz im Zusammenhang mit der Auswahlentscheidung für ein Beratungsangebot jedoch sehr unterschiedlich ausfallen, zeigt die grafische Aufbereitung der Antworten in Abb. 6, wobei die eigene Unternehmensgröße kein relevanter Faktor ist.

Viele Klienten betrachten Vorgehensmodelle als grundlegendes Handwerkszeug der Berater. Sie bilden lediglich die Basis für gute Beratungsleistungen und stellen aus Sicht der meisten Beratungshäuser kein Alleinstellungsmerkmal dar. Ein Interviewpartner vertrat sogar die Meinung, dass Vorgehensmodelle an sich überbewertet werden, da sie trotz ihrer mitunter sehr detaillierten Beschreibungen schlussendlich doch nur einer generischen Vorgehensweise entsprechen. Viele der Befragten teilten die Ansicht, dass Erfahrungen, Referenzen, der Preis sowie die Reputation des Beratungshauses wichtigere Kriterien für die Beraterauswahl sind. 


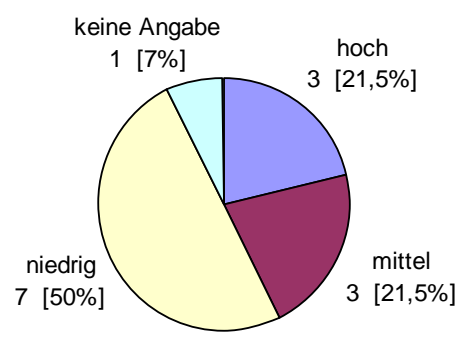

\section{Abbildung 6: Relevanz der Vorgehensmodelle für die Akquisition ( $\mathrm{n}=14)$}

Drei Interviewpartner hingegen maßen den Vorgehensmodellen einen hohen Stellenwert im Rahmen der Projektgewinnung bei. Aus Gründen der Investitions- und Prozesssicherheit fordern die Klienten explizit eine strukturierte und nachvollziehbare Arbeitsweise. Diesbezüglich berichtete ein Berater eines großen Unternehmens, dass das Modell schon in mehreren Projekten ausschlaggebendes Entscheidungskriterium war. Ein anderer Interviewpartner, ebenfalls aus einer großen Beratungsfirma, meinte: „Bei größeren Projekten ist es fast schon ein Ausschlusskriterium, wenn man nicht mit einer standardisierten Methode arbeitet." Aber auch ein selbstständiger Berater wurde schon mehrfach aufgrund seines Vorgehensmodells engagiert und bestätigte die hohe Relevanz des Modells sowohl aus Sicht der Klienten als auch aus eigener Perspektive. Schließlich trägt ein erfolgreiches Vorgehensmodell langfristig zur Steigerung der Reputation des Beratungshauses bei, was wiederum Klienten positiv in ihrer Entscheidung beeinflussen kann.

\subsubsection{Publikation der Vorgehensmodelle}

Die Publikation des eigenen Vorgehensmodells in Zeitschriften- und Buchbeiträgen, auf der eigenen Internetseite oder in Kundenflyern, könnte grundsätzlich die Projektgewinnung fördern, da so Beratungsqualität dargestellt und der Bekanntheitsgrad des eigenen Unternehmens gesteigert werden kann. Dennoch gaben neun (64\%) unserer Interviewpartner an, dass die eigenen Vorgehensmodelle nicht publiziert wurden (Abb. 7). Die Gründe dafür sind allerdings sehr verschieden.

So verzichtet ein Beratungshaus deshalb auf Veröffentlichungen, weil der öffentliche Standard, an den sich das Vorgehensmodell anlehnt, bereits von der diesen Standard entwickelnden Organisation publiziert wird. Andere Gesprächspartner sahen in einer Publikation keinen Mehrwert für die Klientengewinnung. Schließlich ist der Schutz des eigenen Wissens und der Erfahrungen, die in das Vorgehen eingeflossen sind, ein häufiger Beweggrund, die Modelle nicht zu publizieren. Insbesondere die best practices, Templates oder auch einzelne Modellteile werden in diesen Fällen als Wettbewerbsvorteil eingeschätzt und sollen vor der Konkurrenz ,geheim“ gehalten werden. 


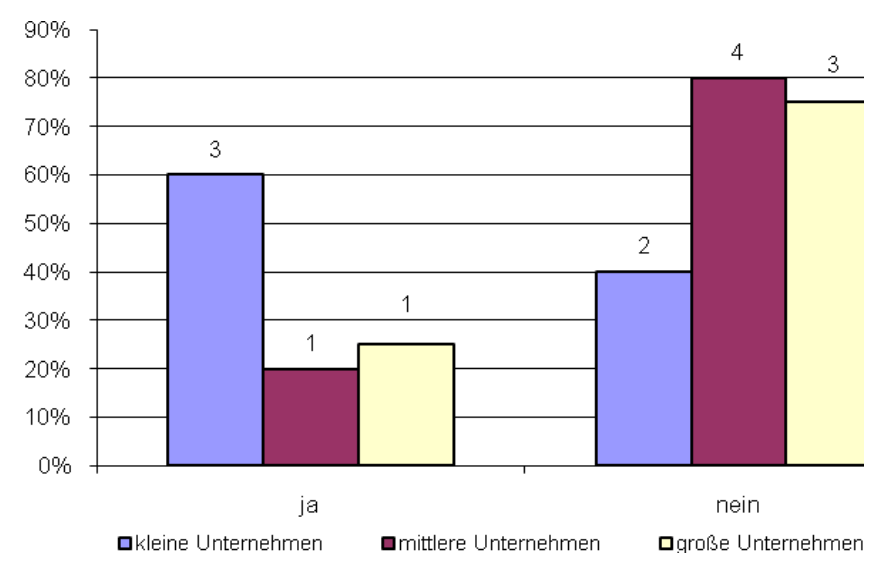

Abbildung 7: Publikation der Vorgehensmodelle $(n=14)$

Die mittelgroßen und großen IV-Beratungshäuser verfolgen eine restriktivere Kommunikationspolitik hinsichtlich ihrer Vorgehensmodelle als die kleinen Beratungen. Ein Grund könnte sein, dass es für die kleinen Unternehmen von vornherein schwerer ist, neue Kontakte zu potentiellen Klienten herzustellen und die Publikation ihrer Vorgehensmodelle hier eine zusätzliche Chance bietet.

\section{$5 \quad$ Fazit}

Die hier vorgestellte Untersuchung zeigt für die beiden klassischen IV-Beratungsfelder der Auswahl und Einführung von Unternehmenssoftware eine überwiegend bereits langjährige Verwendung von Vorgehensmodellen in der Beratungspraxis auf. Diese Modelle entstammen wesentlich eigenen Projekterfahrungen der jeweiligen Beratungshäuser, wobei oft Anregungen aus externen Quellen, wie beispielsweise Industriestandards und Literaturquellen einfließen. Qualitäts- und Effizienzziele sind die dominierenden Gründe für eine Modellentwicklung.

Hinsichtlich der Modellhandhabung ist festzuhalten, dass gerade größere Firmen stark auf die Anwendung der Modelle in Projekten drängen, diese vollständig intern, jedoch nicht nach außen explizieren und dann im Rahmen des Wissensmanagements breit nutzen. Die Modelle sind nur selten Teil einer formalen Zertifizierung gewesen, werden aber regelmäßig intern evaluiert und weiterentwickelt. Ihre Relevanz für die Projektakquisition wird überwiegend als mäßig eingeschätzt, denn sie erfüllen eher eine Erwartung der Klienten hinsichtlich professioneller Beratung als das sie ein wettbewerbsdifferenzierender Faktor wären.

Wegen der methodenbedingt relativ kleinen hier untersuchten Stichprobe sollten die Ergebnisse als erster Hinweis zum status quo gewertet werden, den es in einer nachfolgenden großzahligen standardisierten Befragung zu überprüfen gilt. 


\section{Literatur}

Becker J, Vering O, Winkelmann A (2007) Unternehmenssoftwareeinführung: eine strategische Entscheidung. In: Becker J, Vering O, Winkelmann A (Hrsg.): Softwareauswahl und -einführung in Industrie und Handel, Springer, Berlin und Heidelberg, S. 1-30.

Bundesverband Deutscher Unternehmensberater (2009) Facts \& Figures zum Beratermarkt 2008/9. Marktstudie, Bonn 2009.

Bruhn M (2008) Qualitätsmanagement für Dienstleistungen - Grundlagen, Konzepte, Methoden. 7. Aufl.. Springer, Berlin.

Capgemini (2008) Studie IT-Trends 2008. http://www.de.capgemini.com /m/de/tl/IT-Trends_2008.pdf, 2008-03, Abruf am 2009-09-28.

Gabriel H, Lohnert S (2001) Implementierung von Standardsoftware-Lösungen. In: Scheer A-W, Köppen A (Hrsg) Consulting - Wissen für die Strategie-, Prozess- und IT-Beratung. 2. Aufl.. Springer, Berlin, S. 181-210.

Heinrich L, Heinzl A, Roithmayr F (2004) Wirtschaftsinformatik-Lexikon. 7. Aufl.. Oldenbourg, München.

Maister, DH (2003) Managing the Professional Service Firm. Schuster \& Schuster, London.

Mayer HO (2006) Interview und schriftliche Befragung - Entwicklung,

Durchführung und Auswertung. 3. Aufl., Oldenbourg, München.

Mertens P (1997) Integrierte Informationsverarbeitung. In: Mertens P (Haupthrsg) Lexikon der Wirtschaftsinformatik. 3. Aufl.. Springer, Berlin, S. 208-209.

Mieschke L (2004) Strategisches Geschäftsmodell der Informationstechnologieberatung. DUV, Wiesbaden.

Nissen V, Kinne S (2008) IV- und Strategieberatung - eine Gegenüberstellung. In: Loos P, Breitner, M, Deelmann, T (Hrsg) Proceedings der Teilkonferenz „ITBeratung“ der MKWI 2008. Logos, Berlin, S. 89 - 106.

Nissen V, Simon C (2009) Kernaufgaben und Vorgehensmodelle in der IVBeratung. TU Ilmenau, FG Wirtschaftsinformatik für Dienstleistungen. Forschungsberichte zur Unternehmensberatung Nr. 2009-02.

Seibt D (1997a) Aufbau- und Ablaufstrukturen der Informationsverarbeitung. In: Mertens P (Haupthrsg) Lexikon der Wirtschaftsinformatik. 3. Aufl.. Springer, Berlin, S. 42-43.

Seibt D (1997b) Vorgehensmodell. In: Mertens P (Haupthrsg) Lexikon der Wirtschaftsinformatik. 3. Aufl.. Springer, Berlin, S. 431-434. 
Stahlknecht P, Hasenkamp U (2005) Einführung in die Wirtschaftsinformatik. 11. Aufl. Springer, Berlin. 